\title{
Évaluation préliminaire de l'impact d'un stage pilote d'initiation à la gestuelle chirurgicale sur l'acquisition des habiletés techniques de base par les internes de chirurgie de premier semestre
}

\section{Preliminary evaluation of the impact of an experimental surgical training course based on the acquisition of basic technical skills by surgical interns during their first rotation}

Jérémy de TroyeR ${ }^{1,3}$, Philippe AmabILE ${ }^{1,4}$, Stéphane BerdaH ${ }^{1,5, \star}$, Franck Bladou ${ }^{1,2}$ et Gilles KARSENTY ${ }^{1,2}$

1 CERC (Centre d'Enseignement et de Recherche Chirurgicale) Faculté de médecine Marseille Secteur Nord, Université de la Méditerranée, Marseille, France

2 Chirurgie Urologique, Hôpital Sainte-Marguerite, Assistance Publique-Hôpitaux de Marseille, Faculté de médecine, Université de la Méditerranée, 13009 Marseille, France

3 Gynécologie et Obstétrique, Hôpital Nord, Assistance Publique-Hôpitaux de Marseille, Faculté de médecine, Université de la Méditerranée, 13015 Marseille, France

4 Chirurgie Vasculaire, Hôpital de la Timone, Assistance Publique-Hôpitaux de Marseille, Faculté de médecine, Université de la Méditerranée, 13005 Marseille, France

5 Chirurgie Digestive, Hôpital Nord, Assistance Publique-Hôpitaux de Marseille, Faculté de médecine, Université de la Méditerranée, 13015 Marseille, France

Manuscrit reçu le 20 mai 2008; commentaires éditoriaux formulés aux auteurs le 25 novembre 2008; accepté pour publication le 5 mai 2009

Mots clés :

Chirurgie ;

suture;

habileté ;

simulation ;

pédagogie chirurgicale

^ Directeur du CERC
Résumé - Contexte : L'apprentissage de l'habileté technique chirurgicale (HTC) dans le système français de formation en chirurgie repose essentiellement sur l'immersion en situation réelle et le compagnonnage. De nouvelles contraintes pèsent sur l'exercice de la chirurgie dans les centres hospitaliers universitaires et pourraient réduire l'efficience de ce mode unique de transmission de l'HTC. Objectif : Évaluer l'impact sur l'acquisition de gestes chirurgicaux élémentaires (suture, ligature) d'un stage d'initiation à la gestuelle chirurgicale (SIGC) basé sur l'apprentissage et l'entraînement sur modèle inanimé hors bloc opératoire en complément du mode habituel d'apprentissage par immersion compagnonnage. Méthodes : Nous avons comparé lors d'un exercice standardisé de suture et ligature proposé 3 mois après le début du 
Keywords: Surgery; suture; skill; simulation; surgical education premier stage hospitalier, les performances (scores et durée) d'un groupe d'internes premier semestre exposé au SIGC, à celles d'un groupe d'internes premier semestre non exposés. Résultats : La moyenne du score de performance obtenu, était de 23,9 (15-28) pour le groupe exposé contre 17,1 (2-24) pour le groupe non exposé ( $p=0,036)$. Le test de connaissance du matériel était aussi en faveur du groupe exposé. Le temps moyen de suture ne montrait qu'une tendance en faveur du groupe exposé. Conclusion : Nos résultats suggèrent un impact positif et mesurable d'un stage de technique préliminaire en début d'internat de chirurgie sur la rapidité d'acquisition de gestes de base de chirurgie. Ce type d'enseignement basé sur la simulation pourrait être un complément essentiel au principe d'immersion/compagnonnage pour la transmission de l'HTC.

Abstract - Context: In the French surgical education system, technical skill acquirement in surgery is based on real situations immersion and apprenticeship. There are new constraints affecting the surgical practice in academic hospital centres and it could reduce the efficacy of this unique surgical technical skill (STS) teaching approaches. Objective: To evaluate the impact of an experimental training course based on the acquisition of basic surgical skills (suture, ligature) on inanimate models outside the operative room. This training course was done in addition to the usual real situations immersion and apprenticeship learning approaches. Method: During a standardized suture and ligature drill, which was proposed 3 months after the beginning of the first semester, we compared the performances (scores and time) of a group of residents exposed to the training course to those of a group of residents not exposed to the training course. Results: The mean score obtained was 23.9 (15-28) and $17.1(2-24)$ in the exposed group and in the non-exposed group, respectively: $p=0.036$. The score obtained from the test assessing knowledge of surgical equipment was also higher in the exposed group. The mean time to complete the suture drill had a favourable tendency in the exposed group. Conclusion: Our results suggest that there is a positive and measurable impact of a training course given at the beginning of residency in surgery on the early acquisition of basic surgical technical skills. This type of teaching based on simulation should be a valuable complement to the immersion/apprenticeship teaching approaches for STS knowledge transmission.

\section{Introduction}

Epstein place l'habileté technique pour la pratique de l'examen clinique ou la réalisation d'actes diagnostiques et thérapeutiques parmi les dimensions essentielles de la compétence médicale ${ }^{[1]}$. La mâ̂trise gestuelle indispensable à l'accomplissement d'interventions chirurgicales, que nous appellerons «habileté technique chirurgicale» (HTC), est une capacité spécifique de l'exercice de la chirurgie. Paradoxalement l'apprentissage et l'évaluation de l'HTC ne sont pas formalisés dans le dispositif français de formation à la chirurgie. Absente des objectifs des premier et deuxième cycle d'études médicales, l'acquisition de l'HTC se fait presque exclusivement au cours de l'internat de spécialité lors d'interventions réelles, auxquelles l'élève chirurgien (interne) est invité à participer en tant qu'aide de plus en plus actif d'un chirurgien enseignant. Cette « transmission» du savoir-faire par immersion et responsabilité graduelle est communément désignée par le terme de «compagnonnage ». Elle connaît une limitation croissante liée à la fois à l'évolution de la pratique chirurgicale et au fonctionnement du 
système hospitalo-universitaire. À côté de la légitime question éthique quant à la perte de chance individuelle que peut constituer la réalisation, même sous contrôle, de certains gestes par un novice insuffisamment préparé, on peut citer par exemple : a) la pression économique, qui incite à une optimisation constante du temps d'occupation des salles d'opération ; b) la diminution du temps de présence des internes, qui résulte des directives concernant les repos compensateurs obligatoires les lendemains de gardes ; c) l'accélération de l'innovation technique, qui mobilise les chirurgiens enseignants seniors au service de leur propre formation; d) la pression médico-légale, qui conduit à tout mettre en œuvre pour éviter la survenue de complications après une intervention chirurgicale ${ }^{[2,3]}$.

Tous ces facteurs constituent des contraintes voire des obstacles à l'enseignement de l'HTC aux internes ${ }^{[4]}$. Un des moyens proposé pour faciliter les acquisitions techniques élémentaires est de déplacer leur apprentissage en dehors du bloc opératoire et des contraintes qu'il comporte ${ }^{[4]}$ en utilisant des modèles inanimés. Simples, peu onéreux et toujours disponibles ${ }^{[5-7]}$, ces modèles permettent la démonstration, l'entraînement et l'évaluation à partir d'objectifs standardisés ${ }^{[8]}$.

À titre expérimental depuis trois à quatre ans, dans les facultés de médecine de Marseille, Toulouse et Paris, les candidats nouvellement admis en chirurgie à l'issue des épreuves classantes nationales (ECN) bénéficient avant leur prise de fonction d'un stage d'initiation à la gestuelle chirurgicale (SIGC). Des activités d'apprentissage de base, visant le développement d'HTC, sont organisées en utilisant différents types de modèles et la forte satisfaction enregistrée à la fin du stage (de la part des étudiants autant que des enseignants) suggère que ce type enseignement structuré répond à une attente réelle. L'impact sur les acquisitions techniques n'a cependant pas été évalué à ce jour.

C'est l'objectif de ce travail mis en œuvre à la faculté de médecine de Marseille. Nous avons comparé après trois mois de stage hospitalier les performances respectives des internes de premier semestre ayant participé au SIGC avant leur prise de fonction et celles d'internes qui n'y avaient pas participé, lors d'un exercice standardisé de suture et ligature.

\section{Sujets et méthodes}

\section{Le stage d'initiation à la gestuelle chirurgicale (SIGC)}

En novembre 2006, un stage d'initiation à la gestuelle chirurgicale (SIGC) a été proposé à la nouvelle promotion d'internes admis en filière chirurgicale à la faculté de médecine de Marseille à l'issue des ECN (session mai 2006). En février 2007, ce même stage a été proposé à la nouvelle promotion d'internes admis en filière gynécologie-obstétrique à l'issue des ECN (session mai 2006).

L'objectif était de proposer une séance d'exercices pratiques de gestes de base de chirurgie pour les principales disciplines. Une courte séance théorique avait pour but de présenter la discipline abordée et d'expliquer l'objectif pratique de la séance; elle précédait chaque atelier pratique. Le tableau I résume les objectifs par discipline et les moyens utilisés. Le cadre conceptuel auquel s'adosse la planification pédagogique de ce stage est celui du modèle d'aquisition d'une compétence gestuelle selon Fits et Posner ${ }^{[9]}$, qui prévoit que se succèdent une phase dite «cognitive», puis une phase d'assimilation, qui précède une phase de développement de l'autonomie. Par rapport au dispositif classique «d'immersion/compagnonnage », qui est souvent informel et implicite, le stage est l'occasion de mettre en œuvre un «compagnonnage cognitif explicite ${ }^{[10]}$. Deux des six stratégies d'enseignement décrites par Collins en vue d'un compagnonnage cognitif contextualisé sont développées au cours du stage : d'une part, le recours au modèle (modeling), grâce auquel les étudiants se trouvent dans un contexte d'observation d'une personne qui exécute devant eux une tâche professionnelle, selon un protocole de pensée à voix haute; d'autre part, l'entraînement (coaching), qui permet aux étudiants de réaliser une tâche complexe en recevant l'assistance du professeur qui agit, entre 
Tableau I. Objectifs du stage d'initiation à la gestuelle chirurgicale et moyens pédagogiques mis en œuvre.

\begin{tabular}{|c|c|c|}
\hline $\begin{array}{l}1 / 2 \\
\text { journée }\end{array}$ & Programme & Moyens utilisés \\
\hline 1 & $\begin{array}{l}\text { Principes d'asepsie, lavage des mains, habillage, } \\
\text { champage, circulation au bloc opératoire }\end{array}$ & $\begin{array}{l}\text { Cours théoriques ( } 1 \text { heure) } \\
\text { Travaux pratiques autour d'une table opératoire ( } 2 \text { heures) }\end{array}$ \\
\hline 2 & ligatures et suture & $\begin{array}{c}\text { Cours théorique (40 min) } \\
\text { Pratique sur pieds de porc et stands plastiques (4 heures) }\end{array}$ \\
\hline 3 & Principes en chirurgie digestive et urologique & Cours théorique $(1 \mathrm{~h} 30)$ \\
\hline 4 & Initiation chirurgie ouverte et coelioscopique & Pratique sur porcs anesthésiés $(5 \mathrm{~h} 00)$ \\
\hline 5 & Principes en neurochirurgie & $\begin{array}{c}\text { Cours théorique ( } 1 \text { heure) } \\
\text { Pratique sur sujet anatomique ( } 2 \text { heures } 30)\end{array}$ \\
\hline 6 & Principes en chirurgie orthopédique & $\begin{array}{l}\text { Cours théorique ( } 1 \text { heure }) \\
\text { Pratique sur os sec }(3 \text { heures })\end{array}$ \\
\hline 7 & Principes en chirurgie vasculaire & $\begin{array}{l}\text { Cours théorique (1 heure) } \\
\text { Pratique sur sujet anatomique ( } 2 \text { heures } 30 \text { ) }\end{array}$ \\
\hline 8 & Principes en chirurgie thoracique & $\begin{array}{l}\text { Cours théorique ( } 1 \text { heure) } \\
\text { Pratique sur sujet anatomique ( } 2 \text { heures } 30)\end{array}$ \\
\hline 9 & Principes en réanimation et gestes d'urgence & $\begin{array}{c}\text { Cours théorique ( } 1 \text { heure }) \\
\text { Pratique sur mannequins ( } 3 \text { heures) }\end{array}$ \\
\hline 10 & Bilan et perspective & $\begin{array}{c}\text { Discussion ouverte } \\
\text { Scores de satisfaction anonymes }\end{array}$ \\
\hline
\end{tabular}

autres, comme médiateur entre la connaissance et l'étudiant, dans le cadre d'une forme de pratique guidée.

La participation au stage était proposée sur le mode du volontariat lors des choix d'affectations pour le premier semestre de stage hospitalier d'internat. Pour chaque discipline, les organisateurs avaient requis la présence d'un enseignant senior (professeur des universités-chirurgien des hôpitaux) aidé d'un ou plusieurs juniors.

\section{Constitution des groupes}

Le groupe «exposé » était composé des étudiants admis en filière de chirurgie à Marseille à l'issue des ECN de mai 2006. Tous ont bénéficié du stage d'initiation la semaine précédant leur prise de fonction. Ceux d'entre eux qui effectuaient leur premier semestre en ophtalmologie, neurochirurgie ou chirurgie de la tête et du cou ont été exclus de l'évaluation du fait de leur exposition aléatoire aux gestes de suture de base durant leur stage.

Le groupe «non-exposé » était composé des étudiants admis en filière gynécologie-obstétrique à Marseille à l'issue des ECN de mai 2006. Tous ont bénéficié du stage d'initiation trois mois après leur prise de fonction. Seuls ceux d'entre eux qui effectuaient leur premier semestre en chirurgie gynécologique ont été retenus pour l'évaluation, du fait d'une exposition aux gestes de suture de base comparable au groupe exposé. La participation à l'étude était proposée aux internes après avoir expliqué qu'elle était anonyme et n'aurait aucune influence sur les évaluations sommatives du stage hospitalier.

\section{Méthode d'évaluation de l'impact du stage d'initiation à la gestuelle chirurgicale (SIGC)}

Pour évaluer l'impact du SIGC sur l'acquisition de l'HTC pour les gestes de base (sutures ligatures), nous avons comparé les performances obtenues à un 
exercice standardisé d'un groupe d'étudiants exposé au SIGC à celle d'un groupe d'étudiants non-exposé.

Les internes des deux groupes ont bénéficié du dispositif informel d'immersion/compagnonnage durant les trois premiers mois de stage hospitalier; les internes du groupe «exposé » avaient en outre participé au SIGC et notre hypothèse était que les apprentissages réalisés grâce à ce stage préalable devaient avoir une traduction en termes de meilleures performances lors du test d'évaluation. Ce test d'évaluation a été organisé au terme des trois premiers mois d'internat, immédiatement avant le SIGC destiné aux internes de filière gynécologieobstétrique.

L'exercice comportait trois parties et n'avait pas été proposé lors du SIGC (annexes 1 et 2) :

- la première partie était un questionnaire à choix multiple comprenant sept questions cotées chacune sur un point. Ce questionnaire permettait d'évaluer les connaissances théoriques concernant le choix des fils, aiguilles et instruments, en fonction du geste à réaliser ;

- la deuxième partie consistait en un exercice standardisé de suture et ligature évalué indépendamment par deux observateurs (chirurgiens enseignants), à l'aide d'une grille pré-établie dérivée des Objective standardized assessment of technical skill ${ }^{[8]}$ (OSATS). Le score attribué était la moyenne des deux scores attribués par les deux examinateurs ;

- la troisième partie était le chronométrage de l'exercice de suture, effectué à l'insu des sujets.

Les résultats obtenus par les deux groupes ont été comparés par un test de Student non apparié ; le seuil de significativité était fixé à 0,05 .

\section{Résultats}

Seize internes ont pu être évalués, parmi lesquels sept dans le groupe «exposé » sur un total de 11 éligibles et 9 dans le groupe «non-exposé » sur un total de 10 éligibles. Les cinq internes non évalués (quatre «exposés», un «non exposé ») n’ont pu

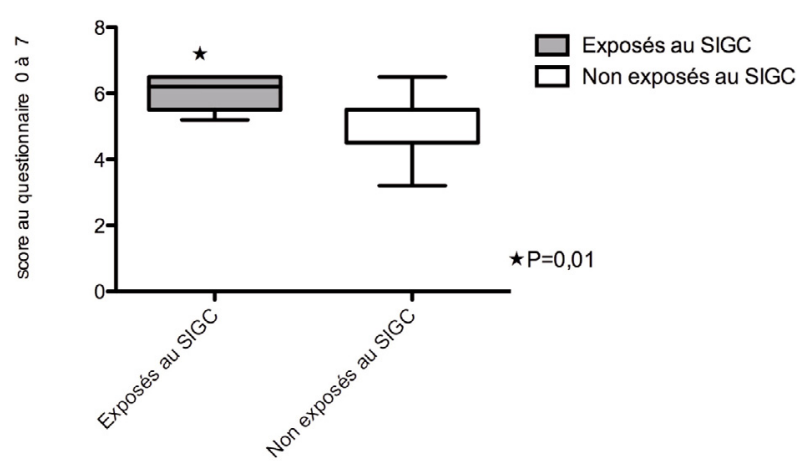

Fig. 1. Performances des internes au questionnaire explorant les connaissances déclaratives relatives au matériel de suture-ligature selon leur exposition préalable au stage d'initiation à la gestuelle chirurgicale (SIGC).

se libérer de leurs obligations hospitalières le jour de l'évaluation. Le SIGC des internes de la filière gynécologie-obstétrique a dû être amputé d'une partie des interventions, la durée du stage n'étant pas compatible avec la continuité des soins des services (impossibilité d'organiser une semaine de stage à temps plein mobilisant tous les internes en premier choix de la filière). Nous avons observé pour le questionnaire et l'exercice de suture une différence significative en faveur du groupe exposé au SIGC. La moyenne des scores obtenu au questionnaire était de $4,9[3,2-6,5]($ score maximum $=7$ ) pour le groupe non exposé vs. 6,04 [5,2-6,5] pour le groupe exposé, $p=0,012$ (figure 1). La moyenne du score obtenu lors de l'exercice de suture était de 17,1 [2-24] (score maximum $=29$ ) pour le groupe non exposé et de 23,9 [15-28] pour le groupe exposé, $p=0,036$ (figure 2). Nous avons observé une différence non significative en faveur d'un temps moyen de suture plus court dans le groupe exposé : 17,3 min [10,3-25] vs. 13,9 $\min [11,1-18,1]$ pour le groupe exposé $(p=0,14)$ (figure 3 ).

\section{Discussion}

Dans le modèle de Fitts et Posner ${ }^{[9]}$ qui vise à décrire l'acquisition d'une compétence gestuelle, la première étape dite cognitive consiste à comprendre chaque étape élémentaire du geste. À ce stade les performances sont médiocres et la réalisation du 


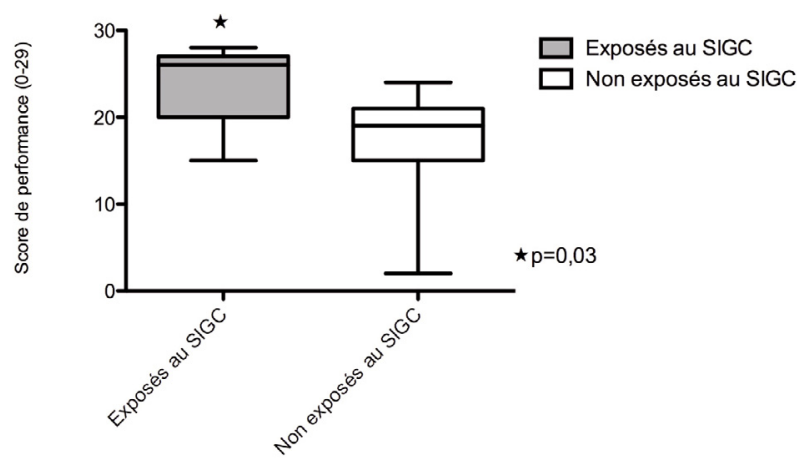

Fig. 2. Performances des internes au test de suture et ligature selon leur exposition préalable au stage d'initiation à la gestuelle chirurgicale (SIGC).

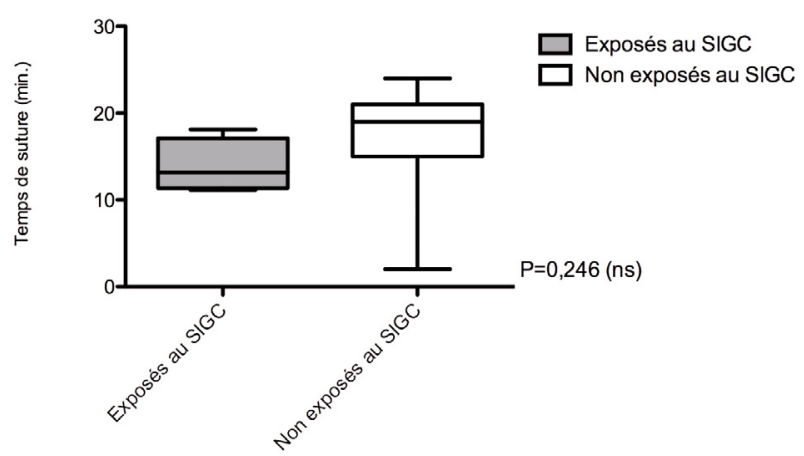

Fig. 3. Performances des internes au chronométrage d'une suture selon leur exposition préalable au stage d'initiation à la gestuelle chirurgicale (SIGC).

geste requiert toute l'attention de l'apprenant. La seconde phase dite d'assimilation permet de passer de la connaissance des étapes élémentaires à un comportement moteur adapté permettant d'enchaîner les étapes élémentaires au prix d'une concentration suffisante. La troisième étape dite d'autonomie, atteinte par l'entraînement, voit l'apprenant automatiser la séquence des étapes élémentaires du geste, tout en allouant ses ressources attentionnelles à un autre objet. Les stations OSATS décrites par Reznick ${ }^{[10]}$ sont construites directement en application de ces principes. L'efficacité de la simulation en chirurgie et du transfert des compétences du modèle à la salle d'opération a principalement été démontré pour l'acquisition des gestes de base chez les débutants ${ }^{[11-13]}$ et ce, quel que soit le type de modèle utilisé $^{[14]}$. Le travail sur modèle inanimé apparaît ainsi comme préparatoire et complémentaire à l'immersion/compagnonnage.

Nos résultats suggèrent une acquisition plus précoce des HTC élémentaires (suture et ligature) durant le premier trimestre de stage hospitalier parmi les internes ayant suivi le SIGC. Bien que des études complémentaires soient nécessaires pour l'établir formellement, il est licite d'admettre l'hypothèse que le SIGC a permis d'amorcer les deux premières étapes du processus d'acquisition (cognition, assimilation) et de sensibiliser l'interne à la nécessité de la troisième (autonomisation). En revanche, la comparaison des temps de suture ne montre pas de différence entre les deux groupes. Il est possible qu'un effet avéré ne soit pas mis en évidence en raison du défaut de puissance de notre étude préliminaire, lié à la faiblesse des effectifs. Il est aussi possible qu'après un seul trimestre de stage, le processus d'automatisation de ces gestes de base ne soit pas achevé. Si elle était confirmée, une telle hypothèse conduirait à recommander de poursuivre la démarche de formation hors bloc au delà du SIGC par un entraînement régulier sur un modèle constamment disponible sur le lieu de stage et/ou à domicile, jusqu'à acquisition évaluée de l'automatisation. Les résultats d'Ericsson ${ }^{[15]}$ qui a montré que la durée de pratique délibérée, c'est-à-dire volontairement et explicitement dédiée, est l'un des déterminants majeurs de haut niveau d'expertise confortent une telle interprétation. La signification des résultats au questionnaire testant des connaissances déclaratives dans le cadre de l'évaluation d'une habileté technique peut être discutée. À notre sens, les connaissances évaluées par ce test (taille, type des aiguilles et des fils par exemple) sont nécessaires à la réalisation avec succès de l'acte technique mais il aurait certainement été plus significatif de les apprécier dans le cadre d'une tâche d'évaluation contextualisée. Il n'est pas possible de dire si le meilleur score observé dans le groupe «exposé » est du à une sensibilisation lors du SIGC ou à une plus forte exposition à ces informations durant leur pratique quotidienne. 
Nous avons constaté, comme le soulignait déjà Heppell $^{[16]}$ en 1995, que les relations étroites et cordiales établies dès le début du cursus entre interne et enseignant au cours du SIGC facilitent l'intégration aux équipes chirurgicales et aide nos internes à acquérir une compréhension plus rapide du milieu complexe que constitue un bloc opératoire. Le SIGC permet enfin de promouvoir une démarche active et curieuse vis-à-vis de l'aspect technique de la pratique chirurgicale. La difficulté d'organisation et l'impossibilité de réunir une promotion complète après le début des choix hospitaliers plaident pour l'organisation d'un SIGC préalablement à la prise de fonction, ce qui lui conférerait en outre la fonction et la valeur d'un dispositif d'accueil. Le développement d'exercices standardisés évaluables et de modèles pertinents pour d'autres compétences techniques que la ligature/suture permettrait d'élargir cette approche de l'enseignement des compétences techniques chirurgicales et de la diffuser à l'ensemble des écoles de chirurgie française.

\section{Conclusion}

Les résultats de cette étude préliminaire montrent qu'un dispositif d'enseignement pratique des habiletés gestuelles de base en chirurgie, développée en recourant à un modèle inanimé en dehors du bloc opératoire en début d'internat, est susceptible d'avoir un impact positif et mesurable sur l'apprentissage de la suture et de la ligature chez les internes de chirurgie de premier semestre. Un enseignement basé sur la simulation apparaît comme un complément utile au principe d'immersion/compagnonnage pour l'acquisition précoce des gestes chirurgicaux de base. Un entraînement régulier dans les mêmes conditions, stimulé par des séances d'évaluation régulière basées sur des objectifs techniques de difficulté croissante, permettrait de prolonger la synergie simulation/immersion durant les premières années de formation chirurgicale.

\section{Contributions}

Jérémy De Troyer a co-rédigé le manuscrit et interprété les résultats. Philippe Amabile a interprété les résultats, complété la recherche bibliographique et participé à la révision du manuscrit. Stéphane Berdah et Franck Bladou ont organisé les stages et participé à la révision du manuscrit. Gilles Karsenty a conçu, co-rédigé le manuscrit, interprété les résultats, effectué la recherche bibliographique et co-organisé les stages.

\section{Annexe 1}

\section{Détail du questionnaire de connaissance du matériel de suture et de l'adaptation matériel-situation}

Q1. L'aiguille la plus pénétrante est :

(Une bonne réponse)
A/ Section triangulaire
B/ Section ronde
C/ A une courbure de 5/8

Q2. Pour passer un point en profondeur dans un espace étroit il vaut mieux une aiguille:

(Une bonne réponse)
A/ Droite
B/ $3 / 8^{\mathrm{e}}$
C/ $4 / 8^{\mathrm{e}}$
D/ $5 / 8^{e}$

Q3. Le fil le plus fin est :

(Une bonne réponse)
A/ Du 10/0
B/ Du $2 / 0$
C/ Du fil tressé 
Q4. Les fils résorbables:

(Une bonne réponse)

A/ Sont dissous ou digérés par le tissus dans lequel ils sont passés

B/ Perdent leur résistance au bout de 10 jours

C/ Sont des fils tressés

Q5. Un porte aiguille se tient:

(Une bonne réponse)

A/ Entre le pouce et l'index

B/ Les doigts solidement enfoncés dans les œillets

C/ Clampé au dernier cran, tenu dans la main sans enfoncer les doigts dans les œillets

D/ Entre le pouce et l'annulaire, la moitié de la $1^{\text {re }}$ phalange dans les œillets

Q6. Une pince à disséquer

(Une bonne réponse)

A/ Se tient dans la main dominante lors de la suture ou de la dissection
B/ Est toujours munie de griffe pour mieux saisir les tissus

C/ A une longueur comparable à celle de l'instrument utilisé dans l'autre main (porte aiguille, ciseaux)

Q7. Le choix d'une pince

à disséquer lors d'une suture

est basée sur

(une ou plusieurs réponses exactes)

A/ La taille du fil que l'on utilise pour suturer

B/ La taille de l'aiguille

C/ La fragilité du tissu que l'on manipule

D/ La profondeur du site opératoire

Total du score obtenu : /7 


\section{Annexe 2}

\section{Évaluation standardisée de l'exercice de suture}

Installation (.../2):

Adapte la hauteur de table (sous les avants bras) :

oui 1 non 0

Oriente la lumière

oui 1 non 0

Choix des instruments (.../9)

Excision :

Bistouri froid lame $23+$ manche 2

Bistouri froid lame $11+$ manche 1

Autre 0

\section{Suture}

Porte aiguille court 3

Autre porte aiguille

Pince autre que porte aiguille 0
Pinces à disséquer griffe courte 2

Autre pince à disséquer griffe 1

Pince mousse 0

Pinces à disséquer griffe courte 2

1 Autre pince à disséquer griffe 1

Pince mousse 0

Gestuelle suture (sans tenir compte des nœuds) (.../9)

Rotation poignet 3-1-0

Travail bi manuel 3-1-0

Régularité du pas $3=+/-1 \mathrm{~mm} \quad 1=+/-2$ à $3 \mathrm{~mm} \quad 0 \mathrm{si}>3 \mathrm{~mm}$ ou objectif non atteint

Temps suture (en secondes) Pour les 4 premiers points départ aiguille chargée ${ }^{\text {er }}$ point arrivée aiguille chargée $5^{\text {e }}$ point (stagiaire non informé du chronométrage ........s

Nouds (.../9)

Demi clefs : deux sens 2, un sens 1, ne sait pas 0 .

Solidité des demi clefs : (on soulève le pied avec le nœud) serré 3 , détendu 1 , lâche 0

Double nœud plats oui 1 non 0

Nœud en profondeur

Descendu et serré en 1 seule fois pour chaque boucle 3

Descendu et serré avec 1 à 2 échecs par chaque boucle 1

Pas descendu, bloqué ou cassé 0

Score total......./29 


\section{Références}

1. Epstein R, Hundert E. Defining and assessing professional competence. JAMA 2002;287:226-35.

2. Coxon JP, Pattison SH, Parks JW, Stevenson PK, Kirby RS. Reducing human error in urology: lessons from aviation. BJU Int 2003;91:1-3

3. Dunkin B, Arales GL, Apelgren K, Mellinger JD. Surgical simulation: a current review. Surg Endosc 2007;21:357-66

4. Hamdorf JM, Hall JC. The developpement of undergraduate curricula in surgery: II. General Surgery. ANZ J Surg 2001;71:108-13

5. Hamdorf JM, Hall JC. Acquiring surgical skills. Br J Surg 2000;87:28-37

6. Davies RJ, Hamdorf JM. Surgical skills training and the role of skills centres. BJU Int 2003;91:3-4

7. Beard J, Jolly B, Newble D, Thomas W, Donnely J, Southgate L. Assessing the technical skills of surgical trainees. Br J Surg 2005;92:778-82

8. Martin JA, Regehr G, Reznick R, et al. Objective Structured Assessment of Technical Skill (OSATS) for surgical resident. Br J Surg 1997;84:273-8.

9. Fitts PM, Posner MI. Human performance. Belmont (CA): Brooks/Cole, 1967.

10. Collins A., Brown J S, Newman SE. Cognitive apprenticeship: Teaching the crafts of reading, writing, and mathematics. In : LB Resnick (Ed.), Knowing, learning, and instruction: Essays in honor of Robert Glaser Hillsdale (NJ): Lawrence Erlbaum, 1990:453-94.
11. Reznick R, Regehr G, Macrae H, Martin J, McCulloch W. Testing technical skill via an innovative "bench station" examination. Am J Surg 1997;173:226-30

12. Anastakis DJ, Regehr G, Reznick RK, Cusimano M, Murnaghan J, Brown M, Hutchison C. Assessment of technical skills transfer from the bench training model to the human model. Am J Surg 1999;177:167-70

13. Grober ED, Hamstra SJ, Wanzel KR, Reznick RK, Matsumoto ED, Sidhu RS, Jarvi KA. The educational impact of bench model fidelity on the acquisition of technical skill: the use of clinically relevant outcome measures. Ann Surg 2004;240:374-81

14. Scott DJ, Bergen PC, Rege RV, Laycock R, Tesfay ST, Valentine RJ, Euhus DM, Jeyarajah DR, Thompson WM, Jones DB. Laparoscopic training on bench models: better and more cost effective than operating room experience? J Am Coll Surg 2000;191:272-83

15. Matsumoto ED, Hamstra SJ, Radomski SB, Cusimano MD .The effect of bench model fidelity on endourological skills: a randomized controlled study. J Urol 2002; $167: 1243-7$

16. Ericsson KA. Deliberate practice and the acquisition and maintenance of expert performance in medicine and related domains. Acad Med 2004;79(10 Suppl):S70-81.

17. Heppell J, Beauchamp G, Chollet A. Ten-year experience with a basic technical skills and perioperative management workshop for first-year residents. Can J Surg $1995 ; 38: 27-32$

Correspondance et offprints : Dr Gilles Karsenty, Chirurgie Urologique, Hôpital Sainte-Marguerite, 270 Bd de Sainte Marguerite, 13009 Marseille, France

Mailto : gilles.karsenty@ap-hm.fr 